\title{
Angioplastia con stents liberadores de rapamicina en diabéticos tipo 2: Experiencia del Hospital DIPRECA
}

\author{
Carolina Ruiz B ${ }^{1}$, Mario Delgado D 2 , Carolina Martínez $C^{1}$, \\ Alicia Valdivieso V4a, Carmen G loria Aylwin $\mathrm{H}^{3}$, \\ Carlos Deck $\mathbf{R}^{4}$, Milton Alcaíno $\mathbf{I}^{4}$.
}

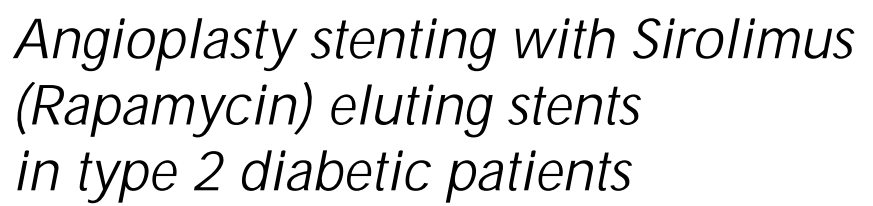

Background: Drug-eluting stents have been developed to reduce the rates of restenosis after coronary angioplasty. Several studies have demonstrated that rapamycin eluting stents are reliable and effective. Aim: To report the experience in our Health Centre with rapamycin-eluting stents. Patients and methods: Forty two stents with rapamicine were implanted to 32 diabetic patients, between June 2002 and December 2004. After the procedure, subjects were clinically followed-up for an average period of $19.9 \pm 9.9$ months, evaluating functional capacity, angina pectoris, dyspnea, need for hospital admission, acute coronary events and cardiac death. In those subjects clinically suspected to have restenosis, a coronary angiography was performed. Results: Twenty-nine subjects (90.6\%) remained asymptomatic, two subjects (6.3\%) developed angina pectoris but restenosis was ruled out, and one subject (3.1\%) died. Conclusions: The use of rapamycin-eluting stents in these patients was safe and successful with no evidence of clinic restenosis. These positive results are similar to those reported in the Diabetes Study (Rev Méd Chile 2007; 135: 573-9).

(Key words: Angioplasty; Sirolimus; Stents)

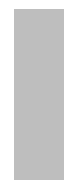

\footnotetext{
Recibido el 9 de marzo, 2006. Aceptado el 23 de octubre, 2006.

${ }^{1}$ Servicio de Medicina Interna, Hospital DIPRECA. ${ }^{2}$ Magíster Salud Pública, Universidad de Chile. ${ }^{3}$ Unidad de Diabetes, Servicio de Medicina Interna, Hospital DIPRECA. ${ }^{4}$ Unidad de Hemodinamia, Centro Cardiovascular, Hospital DIPRECA, Santiago de Chile.

aTecnólogo Médico
}

$\mathrm{L}$ a cardiopatía coronaria es una de las principales causas de morbilidad de la diabetes mellitus tipo 2 (DM 2), y la principal causa de mortalidad, estimándose que 50\% o más de estos

Correspondencia a: Milton Alcaíno I. Unidad de Hemodinamia, Hospital DIPRECA. Av. Vital Apoquindo 1200, Santiago, Chile. Teléfono: 2401118 . Fax: 2431697.

E mail: miltonalc@hotmail.com pacientes fallecen por enfermedad coronaria ${ }^{1}$. El riesgo de los diabéticos tipo 2 de presentar esta patología es 2 a 4 veces mayor que el de la población general, siendo ésta más precoz y severa ${ }^{1}$.

A nivel mundial, hay, aproximadamente, 150 millones de adultos diabéticos, correspondiendo el 95\% a DM2 ${ }^{1}$. En nuestro país, 5\% de la población presenta esta patología ${ }^{2}$. De lo anterior se comprende que la cardiopatía coronaria en el 
paciente diabético, es un problema de salud pública nacional y mundial.

En las últimas 2 décadas, se han producido importantes avances en el tratamiento médico, intervencional y quirúrgico de la enfermedad coronaria, pero los resultados de estas nuevas terapias han sido inferiores en los diabéticos tipo $2^{3,4}$. En relación al tratamiento intervencional, estos pacientes presentan más reestenosis que la población general $^{5}$, manteniendo estos resultados inferiores a pesar del uso de stents ${ }^{6,7}$. Los diabéticos tipo 2, pueden tener 50\% o más de reestenosis angiográfi$\mathrm{ca}^{7,8}$, por lo que se ha establecido que esta patología es un factor de riesgo mayor e independiente para el desarrollo de esta complicación ${ }^{8}$.

La reestenosis se produce fundamentalmente dentro de los 6 primeros meses postangioplastia, especialmente dentro de las primeras semanas ${ }^{8}$. Este fenómeno es secundario a hiperplasia neointimal, proceso que ocurre en forma exagerada en los diabéticos ${ }^{7}$. La reestenosis se define angiográficamente como una disminución del diámetro de la lesión tratada de 50\% o más especialmente dentro de los 6 meses postprocedimiento ${ }^{8}$. Clínicamente, la reestenosis se define como la necesidad de revascularizar la lesión por la aparición de síntomas $^{8}$. Se ha determinado que la reestenosis clínica es aproximadamente la mitad de la angiográfica? 9 . Durante los últimos años, se realizaron varios intentos terapéuticos para disminuir este problema, con resultados negativos ${ }^{10}$, hasta la introducción de stents liberadores de drogas que inhiben la hiperplasia neointimal ${ }^{8}$. Dentro de estas drogas, destaca la rapamicina y el paclitacel, sustancias con las que se tiene la mayor experiencia clínica hasta el momento. Ambas drogas tienen propiedades antimitóticas y antiinflamatorias ${ }^{3-8}$.

La rapamicina o sirolimus es un compuesto originario de Isla de Pascua, que actúa inhibiendo la proliferación de linfocitos y de células musculares lisas, tanto mediada por citoquinas como por factores de crecimiento ${ }^{10}$. Esta sustancia frena el ciclo celular, evitando el paso de G1 (etapa de reposo celular) a $\mathrm{S}$ (etapa de proliferación), inhibiendo así la hiperplasia neointimal ${ }^{8}$.

Varios estudios han demostrado que los stents liberadores de sirolimus son seguros y eficaces en la prevención de reestenosis en la población general y en diabéticos ${ }^{10-13}$. Hasta el momento, sólo se ha realizado un estudio que ha comparado stents liberadores de rapamicina y convencionales en diabéticos tipo $2^{14}$, confirmándose los resultados positivos para el uso de estos nuevos dispositivos. Pero, a pesar de los resultados favorables de estos estudios, todavía no hay claridad absoluta respecto al uso de stents liberadores de drogas en diabéticos tipo $2^{3-15}$, que tradicionalmente son el grupo de pacientes con los peores resultados, tanto con tratamiento médico, intervencional o quirúrgico ${ }^{1}$.

En nuestro centro, entre junio de 2002 y diciembre de 2004, se implantaron 42 stents con rapamicina en 32 diabéticos tipo 2. Los objetivos de este estudio son describir la evolución clínica de estos pacientes y comparar los resultados obtenidos con los descritos en la literatura.

\section{PACIENTES Y MÉTODOS}

Entre junio de 2002 y diciembre de 2004, en nuestro hospital se implantaron 42 stents con rapamicina a 32 diabéticos tipo 2. Se optó por este tratamiento en 28 pacientes, ya que además de ser diabéticos, presentaban otro factor de alto riesgo de reestenosis (lesiones con diámetro menor a $2,7 \mathrm{~mm}$, largo mayor a $20 \mathrm{~mm}$, lesión proximal de la arteria descendente anterior y enfermedad multivaso). Los pacientes estuvieron de acuerdo con recibir este nuevo tipo de stent. Cuatro pacientes sólo presentaban DM 2 como factor de riesgo, pero se consideró su opción personal por optar por este tratamiento.

Las angioplastias se realizaron de acuerdo a las técnicas estandarizadas. El uso de balón de alta presión poststent dependió del criterio del médico operador. Todos los pacientes recibieron aspirina (por lo menos $100 \mathrm{mg} 12 \mathrm{~h}$ previo al procedimiento), clopidogrel (dosis de carga $300 \mathrm{mg}$ el día del procedimiento) y heparina endovenosa durante el procedimiento. Tres pacientes $(10,3 \%)$ recibieron inhibidores IIb/IIIa intraprocedimiento, según criterio del operador. La aspirina se ha mantenido indefinidamente y el clopidogrel, por lo menos, por 6 meses.

El total de la muestra fue seguida clínicamente por un promedio de 19,9 $\pm 9,87$ meses (junio de 2002 a septiembre de 2005), con un mínimo de 9 meses. Se evaluó capacidad funcional, angor, disnea, hospitalizaciones y eventos cardíacos adversos (concepto que incluye síndromes corona- 
rios agudos, muerte de origen cardiaca y necesidad de revascularizar la lesión por síntomas o coronariografía que demuestre reestenosis). En aquellos con sospecha clínica de reestenosis (pacientes con angor, eventos coronarios agudos y deterioro en capacidad funcional), se practicó una coronariografía. Además, se compararon los resultados obtenidos, con los resultados clínicos del único estudio aleatorizado que hasta el momento ha evaluado stents liberadores de rapamicina $y$ convencionales en DM2 ${ }^{14}$.

Para la descripción de nuestra muestra, se utilizó estadística básica (media aritmética, DS y porcentajes). Para comparar nuestros resultados con los descritos en la literatura, se utilizaron la prueba del chi cuadrado y la de Fisher para los valores porcentuales, y la $\mathrm{T}$ de Student para el cálculo de diferencias de valores promedio. El nivel de significación estadística se fijó en 0,05.

\section{Resultados}

Características de los pacientes. Se estudiaron 32 pacientes, 21 hombres $(65,6 \%)$ y 11 mujeres, con una edad promedio de $62,7 \pm 9,3$ años y $8,8 \pm 6,23$ años de diagnóstico de DM2. La mayoría de estos pacientes (69\%) fueron sometidos a angioplastia por angor inestable (Figura 1), presentando en $71,8 \%$ enfermedad multivaso (Tabla 1). El largo promedio de las lesiones fue de $20,4 \pm 7,9 \mathrm{~mm}$, y el diámetro de

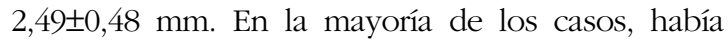
disfunción ventricular izquierda, con una fracción de eyección promedio de $48,6 \pm 14,8 \%$ (medida por ecocardiografía y ventriculografía angiográfica).

Veinticuatro pacientes $(75 \%)$ presentaban otro factor de alto riesgo de reestenosis además de la DM2. Veintidós pacientes $(68,7 \%)$ tenían lesiones con largo mayor a $20 \mathrm{~mm}$ o un diámetro menor a $2,7 \mathrm{~mm}$ y 6 pacientes tenían una lesión proximal de la arteria descendente anterior (4 además con lesiones severas). Dos pacientes presentaban una reestenosis de un stent previo.

En un total de 39 lesiones, se implantaron 42 stents con rapamicina $(1,31 \pm 0,72$ stent por pacien-

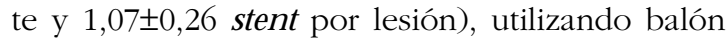
de alta presión poststent en 24 lesiones (61,5\%). Veintidós lesiones $(56,3 \%)$ eran de riesgo moderado a alto de presentar complicaciones agudas postangioplastia (lesiones $\mathrm{B} 2 \mathrm{y} \mathrm{C}$ ). El procedimiento fue exitoso en todos los pacientes (flujo TIMI 3), sin complicaciones intraprocedimiento ni intrahospitalarias. En la Figura 2 se muestra la distribución de los stents.

Seguimiento clínico. Durante el período de observación, 29 pacientes $(90,6 \%)$ se mantuvieron asintomáticos, sin angor y en capacidad funcional I. Dos pacientes $(6,25 \%)$ presentaron angor, el primero a los 6 meses de instalación del stent, y segundo al año de instalación. A ambos pacientes se les practicó una coronariografía, descartándose una reestenosis (Figura 3). Un paciente $(3,12 \%)$ falleció a los 3 meses postangioplastia. Era un hombre de 55 años, portador de una cardiopatía coronaria en fase dilatada y

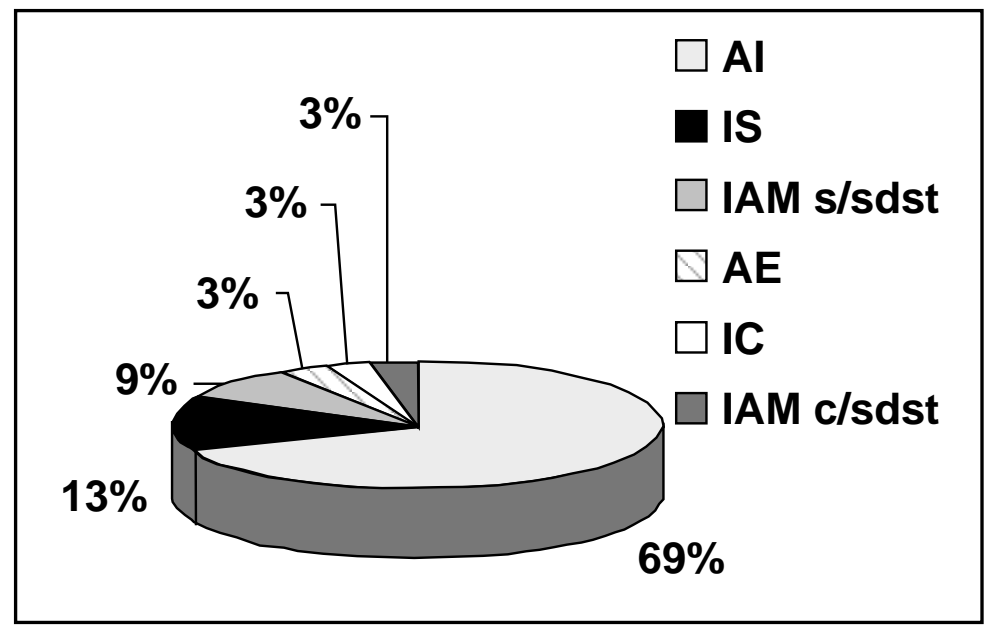

Figura 1. Cuadro clínico al momento de la angioplastia. AI (angina inestable), IS (isquemia silente), IAM S/SDST (infarto sin supradesnivel del ST), AE (angor de esfuerzo), IC (insuficiencia cardiaca), IAM C/SDST (infarto con supradesnivel del ST). 
Tabla 1. C aracterísticas clínicas y angiográficas

\begin{tabular}{|lrr|}
\hline Características & $\mathrm{n}$ pacientes & $\%$ \\
\hline SCA previo & 14 & 43,7 \\
ICP previa & 5 & 15,6 \\
CRM previa & 4 & 12,5 \\
Hipertensión & 24 & 75 \\
Dislipidemia & 22 & 68,7 \\
Obesidad & 10 & 31,2 \\
Tabaquismo & 15 & 46,8 \\
IRC & 6 & 18,7 \\
Tratamiento DM2 & & \\
Régimen exclusivo & 11 & 34,3 \\
ADO & 16 & 50 \\
Insulina & 5 & 15,6 \\
Enf. 1 vaso & 9 & 28 \\
Enf. 2 vasos & 12 & 38 \\
Enf. 3 vasos & 7 & 22 \\
TCI & 2 & 6 \\
PS & 2 & 6 \\
Enfermedad multivaso & 23 & 71,8 \\
Tipo de lesión ${ }^{2}$ (39 lesiones) & & \\
A & 4 & 10,2 \\
B2 & 13 & 33,3 \\
C & 9 & 23 \\
\hline
\end{tabular}

SCA (síndrome coronario agudo), ICP (intervención coronaria percutánea), CRM (cirugía de revascularización miocárdica), IRC (insuficiencia renal crónica), ADO (antidiabéticos orales), TCI (tronco de la coronaria izquierda), PS (puentes safenos de cirugía de revascularización previa).

${ }^{1}$ Insuficiencia renal crónica definida como creatinina $>2 \mathrm{mg} / \mathrm{dl}$ previo al procedimiento. Tres de estos pacientes se encontraban en hemodiálisis crónica.

${ }^{2}$ Clasificación angiográfica de riesgo de complicaciones agudas postprocedimiento (Americam Collage of Cardiology). Las lesiones $\mathrm{B} 2$ y C son de riesgo moderado y alto.

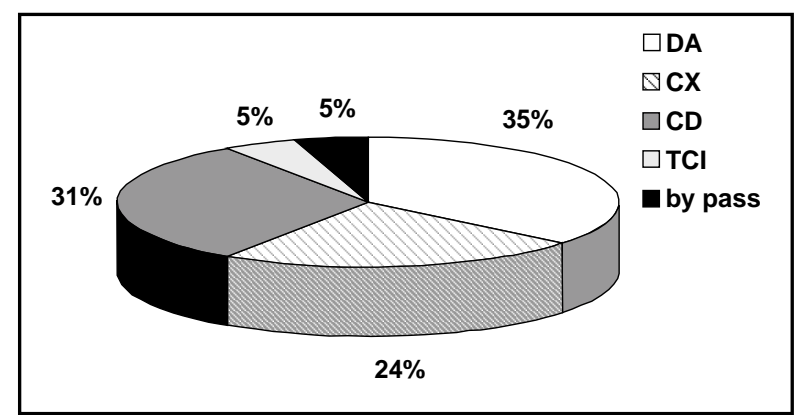

Figura 2. Distribución de los stents. DA (arteria descendente anterior, 15 stents), CD (arteria coronaria derecha, 13 stents), CX (arteria circunfleja, 10 stents), TCI (tronco de la coronaria izquierda, 2 stents), bypass (puentes safenos, 2 stents). 


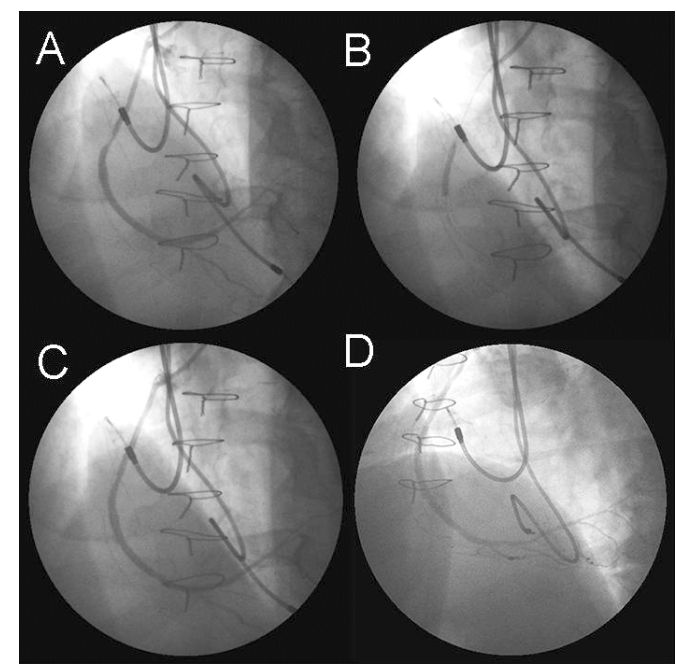

Figura 3. Coronariografía realizada por angor CF II. Lesión severa en puente de vena safena a ACD (jul/2002). B. Instalación stent liberador de rapamicina. C. Flujo TIMI III posprocedimiento. D. Coronariografía julio/04, realizada por angor en CF II, descartó reestenosis.

una disfunción sistólica severa (fracción de eyección 25\%). Además, presentaba una insuficiencia renal crónica en hemodiálisis. Este paciente tenía una lesión severa de la arteria descendente anterior proximal y una oclusión de la arteria coronaria derecha. Se optó por un stent con rapamicina en la arteria descendente anterior dado su alto riesgo quirúrgico. El paciente falleció en su domicilio con un cuadro sugerente de trombosis tardía del stent.

De los 5 pacientes usuarios de insulina de nuestro grupo, un paciente presentó angor al año del procedimiento y los 4 restantes permanecieron asintomáticos.

Comparación de nuestros resultados con los descritos en la literatura. Se realizó una comparación de nuestros resultados, con los resultados clínicos de los 80 pacientes tratados con stents liberadores de rapamicina del estudio Diabetes ${ }^{14}$. Los pacientes de este estudio, fueron seguidos por 9 meses, correspondiendo al mínimo período de observación de nuestra muestra. En la Tabla 2 se muestran las características clínicas y angiográficas de ambos grupos. En la Tabla 3 se muestra la evolución clínica a los 9 meses de seguimiento.

\section{DisCUSIÓN}

Nuestra muestra constituye un grupo con alto riesgo de reestenosis. Además de ser diabéticos, son pacientes que tienen una comorbilidad elevada y varias características angiográficas de mayor riesgo para esta complicación. El 68,7\% de los pacientes tenía lesiones severas y $71,8 \%$ presentaba enfermedad multivaso. Sólo considerando las medidas de referencias de estos pacientes diabéticos, aproximadamente $40 \%$ de ellos debieron presentar reestenosis angiográfica ${ }^{8}$ y $20 \%$ reestenosis clínica?, lo que se traduce en que 5 a 6 pacientes debieron haber sido revascularizados por presentar síntomas. En los pacientes con sospecha clínica de reestenosis, se descartó esta complicación mediante una coronariografía. Consideramos que el paciente fallecido, probablemente presentó una trombosis tardía (trombosis intrastent después de los 30 días), correspondiendo al único evento mayor adverso de nuestro grupo. Los pacientes usuarios de insulina, tuvieron una evolución clínica similar a la del resto de la muestra.

En relación a lo comunicado por la literatura, la muestra del estudio Diabetes ${ }^{14}$ presenta un mayor porcentaje de pacientes que se manejaban con insulina, pero sin diferencia estadísticamente 
Tabla 2. C aracterísticas clínicas y angiográficas de nuestra muestra versus el Estudio D iabetes

\begin{tabular}{|c|c|c|c|}
\hline Características & $\begin{array}{c}\text { RCT DIABETES } \\
\text { (80 pctes con } 111 \text { lesiones) }\end{array}$ & $\begin{array}{c}\text { DIPRECA } \\
\text { (32 pctes con } 39 \text { lesiones) }\end{array}$ & $\mathrm{P}$ \\
\hline Edad & $65,9 \pm 8,8$ & $62,7 \pm 9,3$ & $N s^{* * * *}$ \\
\hline Hombres & $50(62,5 \%)$ & $21(65,6 \%)$ & $\mathrm{Ns}^{*}$ \\
\hline DM2 IR & $26(32,5 \%)$ & $5(15,6 \%)$ & $\mathrm{Ns}^{*}$ \\
\hline DM2 NIR & $54(67,5 \%)$ & $27(84,4 \%)$ & $\mathrm{Ns}^{*}$ \\
\hline Hipertensión & $53(66,3 \%)$ & $24(75 \%)$ & $\mathrm{Ns}^{*}$ \\
\hline Tabaquismo & $36(45 \%)$ & $15(46,8 \%)$ & $\mathrm{Ns}^{*}$ \\
\hline Dislipidemia & $49(61,3 \%)$ & $22(68,7 \%)$ & $\mathrm{Ns}^{*}$ \\
\hline SCA previo & $25(31,3 \%)$ & $14(43,7 \%)$ & $\mathrm{Ns}^{*}$ \\
\hline IPC & $14(17,5 \%)$ & $5(15,6 \%)$ & $\mathrm{Ns}^{*}$ \\
\hline CRM previa & $2(2,5 \%)$ & $4(12,5 \%)$ & $0,05^{* *}$ \\
\hline Enf, Multivaso & $49(61,3 \%)$ & $23(71,8 \%)$ & $\mathrm{Ns}^{*}$ \\
\hline $\mathrm{FE}$ & $66,9 \% \pm 13,1$ & $48,6 \% \pm 14,8$ & $<0,0001^{* * * * *}$ \\
\hline Largo lesión (mm) & $14,5 \pm 8,2$ & $20,4 \pm 7,9$ & $0,0001^{* * * *}$ \\
\hline Diámetro (mm) & $2,33 \pm 0,5$ & $2,49 \pm 0,48$ & $\mathrm{Ns}^{* * * *}$ \\
\hline
\end{tabular}

${ }^{*}$ Chi cuadrado ${ }^{* *}$ Fisher ${ }^{* * *}$ T Student.

DM2 IR (diabetes mellitus 2 insulino requirente), DM2 NIR (diabetes mellitus 2 no insulino requirente), SCA (síndrome coronario agudo), ICP (intervención coronaria percutánea), CRM (cirugía de revascularización miocárdica), FE (fracción de eyección). RCT Diabetes: Randomized comparison trial Diabetes ${ }^{14}$.

Tabla 3. Seguimiento clínico a los 9 meses

\begin{tabular}{|lccc|}
\hline Parámetro & $\begin{array}{c}\text { RCT Diabetes } \\
(\mathrm{n}=80)\end{array}$ & $\begin{array}{c}\text { DIPRECA } \\
(\mathrm{n}=32)\end{array}$ & P \\
\hline Muerte de causa cardiaca & $1(1,3 \%)$ & $1(3,1 \%)$ & $\mathrm{Ns}^{*}$ \\
IAM Q & $1(1,3 \%)$ & 0 & $\mathrm{Ns}^{*}$ \\
IAM no Q & $1(1,3 \%)$ & 0 & $\mathrm{Ns}^{*}$ \\
Lesiones revascularizables $^{1}$ & $6(7,5 \%)$ & 0 & $\mathrm{Ns}^{*}$ \\
Eventos cardíacos adversos $^{2}$ & $9(11,3 \%)$ & $1(3,1 \%)$ & $\mathrm{Ns}^{*}$ \\
\hline
\end{tabular}

*Fisher.

${ }^{1}$ Lesiones que deben revascularizarse por síntomas de isquemia o coronariografía que muestre reestenosis. ${ }^{2}$ Eventos cardíacos adversos. Se consideran IAM, muerte o lesiones revascularizadas.

significativa. Nuestros pacientes presentaban una fracción de eyección más baja y lesiones más largas. En el resto de las características clínicas y angiográficas, ambos grupos son comparables. La evolución clínica de nuestros pacientes ha sido favorable, al igual que lo descrito por el estudio Diabetes ${ }^{14}$. A pesar de las diferencias entre estos grupos, no hay diferencias estadísticamente significativas respecto a la evolución clínica.

Nuestro estudio es retrospectivo, descriptivo y carece de grupo control, por lo que sólo se describe la evolución clínica de la cohorte estudiada en relación a los stents liberadores de rapamicina, sin poder analizar la cuantificación del riesgo o protección atribuible al uso de éstos, o de otras variables como la compensación metabólica o estilos de vida, que pueden haber influido en la evolución de los pacientes.

En conclusión, en nuestra muestra el uso de stents liberadores de rapamicina ha sido exitoso y seguro. Hasta el momento, tras un seguimiento promedio de casi 20 meses, tenemos $0 \%$ de 
reestenosis clínica y 3,1\% de eventos mayores adversos. Dadas las características de nuestra muestra, esperaríamos haber tenido entre 5 a 6 casos de reestenosis clínica. Estos resultados clínicos favorables, concuerdan con los descritos en la literatura, en especial a los del único estudio

\section{REFERENCIAS}

1. Flaherty JD, Davidson CJ. Diabetes and coronary revascularization. JAMA 2005; 293: 1501-8.

2. Carrasco E. Epidemiología de la diabetes mellitus. En: De Los Ríos M, ed. Diabetes Mellitus. $2^{2}$ Ed. Santiago: Editorial Fundación de Investigación y Perfeccionamiento Médico, 2003; 33-41.

3. Moussa I, León MB, Baim DS, O’Neill WW, Popma JJ, Buchinder M ET AL. Impact of sirolimus-eluting stents on outcome in diabetic patients: a SIRIUS (SIRolImUS-coated Bx Velocity balloon-expandable stent in the treatment of patients with de novo coronary artery lesions) substudy. Circulation 2004; 109: 2273-8.

4. West Ne, Ruygrok PN, Disco CM, Webster MW, Lindeboom WK, O'Neill WW et al. Clinical and angiographic predictors of reestenosis after stent deployment in diabetic patients. Circulation 2004; 109: 867-73.

5. Gilbert J, Raboud J, Zinman B. Meta-analysis of the effect of diabetes on reestenosis rates among patients receiving coronary angioplasty stenting. Diabetes Care 2004; 27: 990-4.

6. Eiezi S, Kastrati A, Pache J, Wehinger A, Hadamitzky M, Dirschinger J et al. Diabetes mellitus and the clinical and angiographic outcome after coronary stent placement. J Am Coll Cardiol 1998; 32: 1866-73.

7. Abizaid A, Costa Ma, Blanchard D, Albertal M, Eltchaninoff H, Guagliumi G et al. Sirolimuseluting stents inhibit neointimal hyperplasia in diabetic patients. Insights from the RAVEL Trial. Eur Heart J 2004; 25: 107-12.

8. Moses JW. Drug eluting stents: sirolimus. $9 \mathrm{TH}$ Annual Interventional Cardiology Fellows Course - Transcatheter Cardiovascular Therapeutics 2003. Disponible en www.tctmd.com (consultado en agosto 2005).

9. Serruys PW, van Hout B, Bonnier H, Legrand V, García E, Macaya C et al. Randomized comparison aleatorizado con stents liberadores de rapamicina diseñado para diabéticos tipo $2^{14}$.

Consideramos que los stents liberadores de rapamicina pueden ser una alternativa terapéutica para el manejo de la cardiopatía coronaria en el diabético tipo 2 .

of implantation of heparin-coated stents with balloon angioplasty in selected patients with coronary artery disease (Benestent II). Lancet 1998; 352: 673-81.

10. Morice MC, Serruys PW, Sousa Je, Fajadet J, Ban Hayashi E, Perin M et al. RaVEl Study Group. Randomized Study with the Sirolimus-Coated Bx Velocity Balloon-Expandable Stent in the Treatment of Patients with de Novo Native Coronary Artery Lesions. A randomized comparison of a sirolimus-eluting stent with a standard stent for coronary revascularization. N Engl J Med 2002; 346: $1773-80$.

11. Moses JW, León MB, Popma JJ, Fitzgerald PJ, Holmes DR, O'Shaughnessy C et al. SIRIUS Investigators. Sirolimus-eluting stents versus standard stents in patients with stenosis in a native coronary artery. N Engl J Med 2003; 349: 1315-23.

12. Schampaert E, Cohen EA, Schluter M, Reeves F, Traboulsi M, Title LM et al. C-SIRIUS Investigators. The Canadian study of the sirolimus-eluting stent in the treatment of patients with long de novo lesions in small native coronary arteries (CSIRIUS). J Am Coll Cardiol 2004; 43: 1110-5.

13. Schofer J, Schluter M, Gershlick AH, Wijns W, García E, Schampaert E et al. Sirolimus-eluting stents for treatment of patients with long atherosclerotic lesions in small coronary arteries: doubleblind, randomized controlled trial (E-SIRIUS). Lancet 2003; 362: 1093-9.

14. Sabate M, Jiménez-Quevedo P, Angiolillo DJ, GómezHospital JA, Alfonso F, Hernández-Antolin R et al. Randomized comparison of sirolimus-eluting stent versus standard stent for percutaneous coronary revascularization in diabetic patients: the diabetes and sirolimus-eluting stent (Diabetes) trial. Circulation 2005; 112: 2175-83.

15. Finn AV, Palacios IF, Kastrati A, Gold HK. Drugeluting stents for diabetes mellitus: a rush to judgment? J Am Coll Cardiol 2005; 45: 479-83. 